\title{
A Simple and New Device to Avoid Hepatic Venous Outflow Obstruction in Adult Liver Transplantation
}

\author{
Dino Donataccio $^{1}$, Salvatore Grosso ${ }^{2}$, Matteo Donataccio ${ }^{1 *}$ \\ ${ }^{1}$ Liver Transplant Unit, Azienda Ospedaliera Universitaria Integrata, Verona, Italy \\ ${ }^{2}$ Anesthesia and Resuscitation Unit, Azienda Ospedaliera Universitaria Integrata, Verona, Italy \\ E-mail: "matteodonataccio@ospedaleuniverona.it \\ Received August 30, 2011; revised November 17, 2011; accepted November 30, 2011
}

\begin{abstract}
Hepatic venous drainage in liver transplantation may be reduced to the level of caval anastomosis producing an obstruction degree and leading to serious vascular complication such as the acute Budd-Chiari syndrome, which may result in organ loss. Outflow obstruction may be caused by lack of technique in caval anastomosis or by allograft malposition as a consequence of anatomical graft and recipient conditions. Fixation of the round ligament, placement of bowel loops and use of tissue expanders have been described to stabilize graft position during liver transplantation with related procedure complications. We report our experience of a simple homemade device using a surgical glove expander that allowed us to successfully avoid outflow obstruction in all of nine treated patients. No device related complications occurred. In malposed liver allografts, we strongly suggest the use of this simple and safe device to avoid hepatic venous outflow obstruction on condition that the device is early removed within 48 hours.
\end{abstract}

Keywords: Outflow Obstruction, Allograft Malposition, Liver Transplantation, Surgical Glove Expander, Device

\section{Introduction}

Outflow obstruction in liver transplantation causes graft dysfunction and eventual graft loss. In the allograft, partial mechanical hepatic outflow obstruction is uncommon but not rare. Venous drainage after liver transplantation (LT) may be hindered by the level of caval anastomosis, producing an obstruction degree leading to the most serious acute Budd-Chiari syndrome (B-C) which may lead to organ loss. It may be caused by lack of technique in caval anastomosis construction resulting in a stenosis of anastomosis, and by graft and/or recipient anatomical conditions resulting in an allograft malposition. Acute B$\mathrm{C}$ is an early postoperative vascular complication that accounts for about 3\% of LTs. This complication usually occurs in recipient vena cava preserving techniques, more frequently in piggy-back (PB) technique due to the graft mobility along two orthogonal planes, antero-posterior and latero-lateral (Figure 1). In latero-lateral (L-L) caval anastomosis technique, especially with a wide anastomosis, graft mobility is only allowed along the lateral plane. Thus the caval anastomosis proves to be a sort of hinge which does not allow antero-posterior mobility (Figure 2)
[1,2]. In recipients the presence of a deep fovea hepatis and a wide empty subphrenic space may allow a venous twisting caused by displaced liver graft after repositioning into the abdomen, leading to a consequent hepatic venous outflow obstruction. Another cause may be identified by the donor/recipient dimensional mismatch: a too bulky graft may cause a compression of caval anastomosis during the abdominal incision closure and vice versa a too small graft may rotate towards the hepatic fossa producing a twisting of the caval anastomosis and therefore an hindrance to the venous outflow of the graft. In paediatric split liver transplantation [3] and in living donor liver transplantation [4], the rate of hepatic venous outflow obstruction may be much higher, up to $6.6 \%$ and $9.5 \%$ respectively, due to a venous twisting favored by a greater mobility of a smaller graft.

This form of obstruction should be early recognized and promptly relieved to prevent complications and graft failure. We experienced this complication intraoperatively in nine patients undergoing liver transplant. These patients presented an unexplainable hypotension after portal reperfusion. In all cases, doppler ultrasonography, by determining vascular flow patterns and velocities, was 


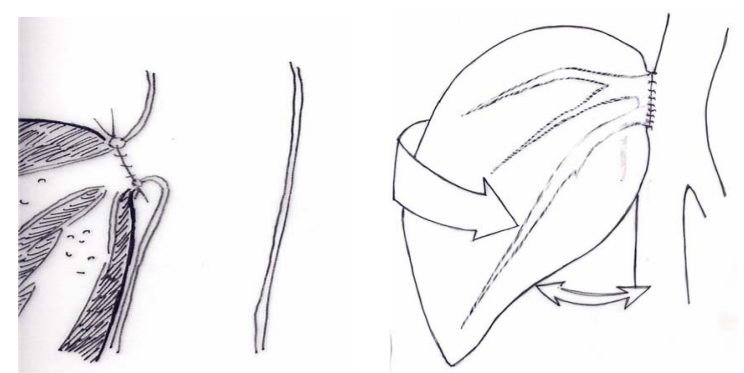

Figure 1. Piggy-Back caval anastomosis.

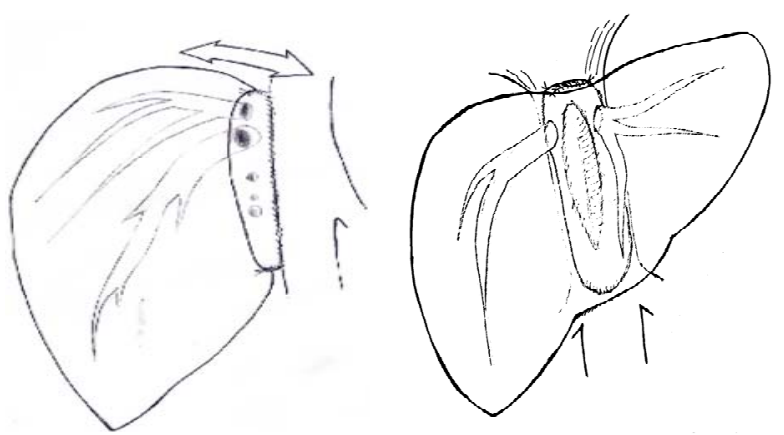

Figure 2. Latero-lateral caval anastomosis.

able to detect the cause of venous outflow obstruction. The use of tissue expanders has been described to stabilize graft position during liver transplantation [5]. In this study, we applied a surgical glove filled up with 200/300 $\mathrm{ml}$ of saline solution to improve hepatic vein outflows. We retrospectively reviewed our experience and analyzed the efficacy and safety of this procedure.

\section{Patients and Methods}

Between December 2002 and January 2011, 142 consecutive adult LTs were performed in 139 patients. Surgical technique was performed by the same experienced surgeon in all patients. Hepatectomy with recipient vena cava preservation and without venous bypass was possible in $100 \%$ of surgical procedures. Caval anastomosis was performed in latero-lateral technique aiming the widest anastomosis possible up to seven centimeter. This was achieved in all but two patients. The first one was a recipient with a double inferior vena cava confluencing at diaphragmatic caval ostium and a termino-lateral anastomosis was constructed; in the other one, with a donor vena cava encircled by caudate lobe, a PB implantation was performed. The donor/recipient body weight mismatch acceptable was up to $30 \%$ in favor of the donor.

In nine cases (6.3\%), a graft venous outflow obstruction was clinically diagnosed after portal reperfusion. The blood pressure fell down due to hypovolemia and a reduced central venous pressure was registered. The graft was congested with a high risk of bleeding. Lifting and rotating to the left side of the graft allowed immediate hemodynamic recovery. Then a surgical glove was filled with 200/300 $\mathrm{ml}$ of saline solution and pushed under the right side of the graft supporting to counteract the venous outflow obstruction (Figure 3). The glove was removed within 48 hours with a very slight reopening under hemodynamic monitoring.

\section{Results}

We experienced venous outflow obstruction in nine cases (6.3\%), seven whole liver and two right split graft. In three cases the caval anastomosis was compressed by a too bulky graft. In the other six cases the graft fell into a too deep fovea hepatis causing an outflow obstruction. Our glove device effectively overcame this complication. In all cases the glove was removed within 48 hours. No venous outflow obstruction has been detected by doppler ultrasound monitoring during and after removal of the glove. No technique related complications have been registered and no graft has been lost due to venous outflow complications. The median follow-up is 32 months (range 3 - 88).

\section{Discussion}

The early venous outflow obstruction is a rare but potentially fatal complication of LT. Despite refinement in surgical technique, mechanical outflow obstructions from malpositioning of the graft may happen. Oftentimes, it mimics hypovolemia because of decreased venous return. Hypotension, as a result of hepatic outflow obstruction, is a temporary hypovolemia. To treat abnormal data from hemodynamic monitoring, blind resuscitation with fluids will not solve the problem. Once detected, outflow blockade must be relieved immediately usually by surgical intervention. Mechanical obstruction from vessel anastomosis, thrombosis, kinking and twisting should be managed surgically by meticulous venous anastomosis and/or adjusting the position of the graft [6]. This can be done by maneuvers such as fixation of the round ligament to

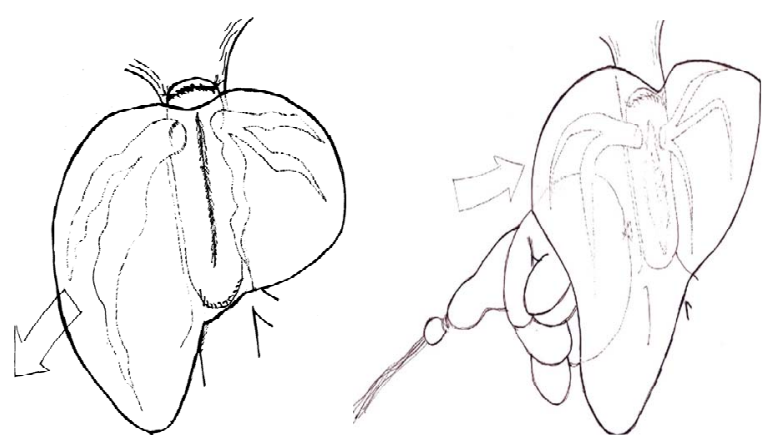

Figure 3. Glove expander. 
fix the graft in position, placement of bowel loop to lift the graft, additional side-to-side cavo-cavostomy [5,7,8], placement of a Blakemore-Sengstaken tube [9] or use of tissue expander $[5,10]$. Conservatively, it may be treated by angioplasty but restenosis is frequent, or by positioning a vascular stent with resolution of clinical symptoms in $73 \%$ to $100 \%$ of cases [11]. The surgical procedure with an additional cavo-caval anastomosis or by retransplantation, entails a high mortality rate $[12,13]$.

In 2005 Wang et al. [14] reported that the use of tissue expander and Foley catheter improved venous inflow and outflow; all expanders were removed within the $19^{\text {th }}$ $56^{\text {th }}$ postoperative day; seven complications occurred in five out of seven treated patients.

All our devices were removed early within 48 postoperative hours without any complications. We were never compelled to reposition the volumetric support. In two cases, a fluid collection in the residual space after device removal persisted several months before reabsorption without any infective complications. No graft has been lost due to venous outflow complications. In our opinion the graft compliance to the recipient subphrenic space is much more than commonly believed and rapidly allows the graft to reach stability, thus overcoming venous outflow obstruction. An early removal of device can avoid related complications.

In conclusion, after liver transplantation in case of intraoperative not suture related venous outflow obstruction, a simple and low cost homemade volumetric support may allow the graft to adapt to the available space and overcome a critical congestion.

\section{References}

[1] P. Parrilla, F. Sánchez-Bueno, J. Figueras, E. Jaurrieta, J. Mir, C. Margarit, J. Lázaro, L. Herrera, M. GómezFleitas, E. Varo, E. Vicente, R. Robles and P. Ramirez, "Analysis of the complications of the Piggy-Back Technique in 1112 Liver Transplants," Transplantation, Vol. 67, No. 9, 1999, pp. 1214-1217. http://dx.doi.org/10.1097/00007890-199905150-00003

[2] F. Navarro, M. C. Le Moine, J. M. Fabre, J. Belghiti, D. Cherqui, R. Adam, F. R. Pruvot, C. Letoublon and J. Domergue, "Specific Vascular Complications of Orthotopic Transplantation with Preservation of the Retrohepatic Vena Cava: Review of 1361 Cases,” Transplantation, Vol. 68, No. 5, 1999, pp. 646-650. http://dx.doi.org/10.1097/00007890-199909150-00009

[3] G. Krishna Kumar, K. Sharif, D. Mayer, D. Mirza, K. Foster, D. Kelly and A. J. Millar, "Hepatic Venous Outflow Obstruction in Paediatric Liver Transplantation," Pediatric Surgery International, Vol. 26, No. 4, 2010, pp. 423-425. http://dx.doi.org/10.1007/s00383-010-2564-y

[4] G. Y. Ko, K. B. Sung, H. K. Yoon, K. R. Kim, J. H. Kim, D. I. Gwon and S. G. Lee, "Early Posttransplant Hepatic
Venous Outflow Obstruction: Long-Term Efficacy of PriMary Stent Placement,” Liver Transplantation, Vol. 14, No. 10, 2008, pp. 1505-1511. http://dx.doi.org/10.1002/lt.21560

[5] Y. Inomata, K. Tanaka, H. Egawa, S. Uemoto, T. Kiuchi, K. Satomura, S. Uyama and H. Okajima, "Application of a Tissue Expander for Stabilizing Graft Position in Living-Related Liver Transplantation," Clinical Transplantation, Vol. 11, No. 1, 1997, pp. 56-59.

[6] B. Jawan, H. K. Cheung, C. C. Chen, Y. S. Chen, Y. C. Chiang, C. C. Wang, Y. F. Cheng, T. L. Huang, H. L. Eng, S. Goto, T. L. Pan, V. De Villa, P. P. Liu, S. H. Wang, C. L. Lin and J. H. Lee, "Repeated Hypotensive Episodes Due to Hepatic Outflow Obstruction during Liver Transplantation in Adult Patients,” Journal of Clinical Anesthesia, Vol. 12, No. 3, 2000, pp. 231-233. http://dx.doi.org/10.1016/S0952-8180(00)00146-X

[7] J. Lerut and P. Gertsch, "Side-To-Side Cavo-Cavostomy: A Useful Aid in "Complicated" Piggy-Back Liver Transplantation,” Transplant International, Vol. 6, No. 5, 1993, pp. 299-301. http://dx.doi.org/10.1007/BF00336033

[8] A. C. Stieber, R. D. Gordon and N. Bassi, “A Simple Solution to a Technical Complication in "Piggyback" Liver Transplantation,” Transplantation, Vol. 64, No. 4, 1997, pp. 654-655.

[9] B. Malassagne, B. Dousset, P. P. Massault, D. Devictor, O. Bernard and D. Houssin, "Intra-Abdominal Sengstaken-Blakemore Tube Placement for Acute Venous Outflow Obstruction in Reduced-Size Liver Transplantation,” British Journal of Surgery, Vol. 83, No.8, 1996, p. 1086. http://dx.doi.org/10.1002/bjs.1800830814

[10] J. C. Emond, T. G. Heffron, P. F. Whitington, C. E. Broelsch, "Reconstruction of the Hepatic Vein in Reduced Size Hepatic Transplantation,” Surgery, Gynecology \& Obstetrics, Vol. 176, No. 1, 1993, pp. 11-17.

[11] M. D. Darcy, "Management of Venous Outflow Complications after Liver Transplantation,” Techniques in Vascular and Interventional Radiology, Vol. 10, No. 3, 2007, pp. 240-245. http://dx.doi.org/10.1053/j.tvir.2007.09.018

[12] J. Quintela, C. Fernàndez, J. Aquirrezabalaga, C. Gerardo, M. Marini, F. Suarez and M. Gomez, "Early Venous Obstruction after Liver Transplantation and Treatment with Cavo-Cavostomy," Transplantation Proceedings, Vol. 41, No. 6, 2009, pp. 2450-2452. http://dx.doi.org/10.1016/j.transproceed.2009.06.066

[13] A. Cavallari, M. Vivarelli, R. Bellusci, E. Jovine, A. Mazziotti and C. Rossi, "Treatment of Vascular Complications Following Liver Transplantation: Multidisciplinary Approach," Hepatogastroenterology, Vol. 48, No. 37, 2001, pp. 179-183.

[14] C. C. Wang, A. M. Concejero, C. C. Yong, Y. S. Chen, S. H. Wang, C. C. Lin, Y. W. Liu, C. H. Yang, T. S. Lin, K. C. Hung, B. Jawan, Y. F. Cheng, S. Ibrahim and C. L. Chen, "Improving Hepatic and Portal Venous Flows Using Tissue Expander and Foley Catheter in Liver Transplantation," Clinical Transplantation, Vol. 20, No. 1, 2006, pp. 81-84.

http://dx.doi.org/10.1111/j.1399-0012.2005.00431.x 